Mitteilungen

\title{
18. Treffen der hauptamtlichen Laborleiter der Krankenhäuser in Nordrhein-Westfalen
}

\author{
Heinz Fritze* \\ Katholisches Klinikum Duisburg, St. Johannes-Hospital, \\ Duisburg, Deutschland
}

Nach traditionellem und bewährtem Muster fand das 18 . Treffen der nordrhein-westfälischen Laborleiter am 8. und 9. Oktober 2004 wiederum in Bochum statt.

Herr Krieg (Bochum) moderierte das Treffen am ersten Tag. Zum Auftakt berichtete Herr Doss (Marburg) über das Thema "Porphyrie-Diagnostik". Durch seine grundlegenden Untersuchungen hat Herr Doss diesen Spezialbereich der Labordiagnostik wesentlich geprägt. In seinem Vortrag gab er einen Überblick über die pathophysiologischen Zusammenhänge bei Porphyrien. Porphyrien sind Krankheiten der Hämbiosynthese, hervorgerufen durch hereditäre oder toxische Störungen. Sieben Enzyme steuern die Hämbiosynthese. Jeder Defekt eines einzelnen Enzyms wirkt durch Akkumulation oder Exkretion einzelner Komponenten des Porphyrinstoffwechsels pathologisch. Anatomisch zeigt sich ein Defekt vorwiegend im Knochenmark (Erythropoese) oder in der Leber. In einer Fülle von Detaildaten wies Herr Doss auf die komplizierten Zusammenhänge in der Pathophysiologie hin. Es kommen hepatische und nichthepatische Formen der Porphyrie vor, akute und nicht akute. 20\% der Porphyrien sind hereditär, der Rest wird durch Enzymmangel bei Stress, Medikamenteneinnahme und unter dem Einfluss anderer toxischer Agenzien wie z.B. Blei ausgelöst. In der Diagnostik kommt die klassisch-moderne Analytik mittels Chromatographie zum Einsatz. Molekularbiologische Methoden sind auf dem Vormarsch. Diagnostisch ist die Porphyrie eine schwierige Erkrankung, da sie interdisziplinär auftritt. Manche Patienten haben eine lange Odyssee von Facharzt zu Facharzt hinter sich, ehe die wahre Ursache der Erkrankung erkannt wird. Herr Doss ist inzwischen emeritiert, aber noch beruflich aktiv (www.porphyrie.de). Es ist zu hoffen, dass er sein Spezialwissen noch lange zum Wohle der Patienten einsetzen kann.

Anschließend informierte Herr Willamowski (Mannheim) über das "Medizinproduktegesetz (MPG) und die CE-

${ }^{*}$ Korrespondenz: Dr. rer. nat. Heinz Fritze, Katholisches Klinikum Duisburg, St. Johannes-Hospital, Zentrallaboratorium, An der Abtei 7-11, 47166 Duisburg, Deutschland
Kennzeichnung". Zunächst beschrieb er die grundlegenden Definitionen der wichtigsten Begriffe im MPG wie Hersteller, Medizinprodukt, in-vitro-Diagnosticum und Zubehör. Die wesentlichen Anforderungen beziehen sich auf die dauerhafte Qualität und die Sicherheit der Produkte. Dafür verantwortlich ist der Hersteller. Er stellt die Konformitätserklärungen aus und beantragt die Zuerkennung der CE-Kennzeichnung. Das CE-Kennzeichen bedeutet demnach Sicherheit, Eignung und Leistung des angebotenen Produktes. Erst mit dieser Kennzeichnung darf der Hersteller sein Produkt in Verkehr bringen. Bei der Anwendung des Produktes ergeben sich für den Anwender im Labor Pflichten, die in der Medizinprodukte-Betreiberverordnung festgelegt worden sind. Vor allen Dingen muss er dafür sorgen, dass das Produkt (Gerät, Reagenz o.ä.) von geschultem Personal in fachlich einwandfreier Weise eingesetzt, betrieben oder verbraucht wird. Hierzu gehören u.a. Schulung, Wartung, interne und externe Qualitätskontrolle und die entsprechende Dokumentation. Von Bedeutung ist die Änderung der Zweckbestimmung. Dieser Fall tritt ein, wenn im Labor Gerät und Reagenz von verschiedenen Herstellern eingesetzt werden. Jetzt geht die Verantwortung für die Konformität auf den Anwender über. Er muss durch entsprechende Validation für die Sicherheit und Konformität der Kombination sorgen. Dieser Punkt warf in der anschließenden Diskussion einige Fragen auf. Welchen Aufwand muss ein Labor leisten, damit es diese Anforderungen erfüllen kann? Scheinbar offene Systeme sind tatsächlich geschlossen. Werden kleine Firmen vom Markt gedrängt?

Nach der Pause sprach Herr Genth (Aachen) über die "Diagnostik von Kollagenosen". Kollagenosen sind entzündlich-rheumatische Erkrankungen, die verschiedene Organe und das Bewegungssystem befallen können. Die Aetiologie der Kollagenosen weist immer auf entzündliche Prozesse auf dem Hintergrund immunologischer Reaktionen hin. Kollagenosen gehen oft einher mit der Bildung von Autoantikörpern, die im Labor bestimmt werden können. Deren Sensitivität und Spezifität sind zwar eingeschränkt, sie können jedoch differentialdiagnostisch und zur Prognoseerstellung eingesetzt werden. Ausschlussdiagnostik ist mit sensitiven Gruppentests gut zu betreiben. Der Nachweis von spezifischen Autoantikörpern ist jedoch recht schwierig. Es gibt wenig Assoziation zwischen der Konzentration und unterschiedlichen Epitopen. Zudem sind die Tests 
methoden- und firmenabhängig. Standards sind nicht vorhanden. Die Bestimmung der antinukleären Antikörper mit HEP2-Zellen und Fluorescein-markierten Antikörpern ist immer noch die Standardmethode. Der besondere Vorteil dieser mikroskopischen Methode liegt in der Beurteilung des Fluoreszenzmusters der Zelle. Diesen Vorteil bieten ELISA-Autoimmunprofile nicht. Die Differenzierung von Antikörpern erfolgt mittels ELISA, Immunpräzipitation und Immundiffusion. Zunehmend werden Dot Blots verwendet, die mehrere Antikörper auf einmal erfassen können. Anschließend beschrieb Herr Genth die Diagnostik einzelner Systemerkrankungen wie SLE (systemischer Lupus erythematodes), systemische Sklerose, idiopathische Myositis und undifferenzierte Kollagenosen.

Zum Tagesabschluss wie traditionell üblich ein Thema, das über den fachspezifischen Tellerrand hinausging. Herr Metzinger (Mainz) behandelte die Frage "Was ist Bewusstsein?".

Die Gehirnforschung dringt immer tiefer in die zellulären und molekularen Funktionsabläufe des Gehirns ein. Das Gehirn ist zum Objekt von Forschung und Manipulation geworden. Dabei ist das Phänomen "Bewusstsein" als zentrale Frage ins Blickfeld gerückt. Was ist Bewusstsein? Dieses Phänomen impliziert ein erkenntnistheoretisches Problem. Die einen beschreiben es aus der Erste-Person-Perspektive (Introspektive), während andere versuchen, objektive Kriterien von außen zu erstellen. Herr Metzinger beklagt, dass moderne Bewusstseinsphilosophen alles zu materialistisch sehen. Sie behaupten, dass eine eigene Innenperspektive nur materiell zu erklären sei. Denken, Fühlen und Handeln werden durch neuronale Netzstrukturen bestimmt. Experimente zeigen, dass bestimmte Handlungen konkreten Hirnregionen zugeordnet werden können. Der propagierte Paradigmenwechsel lässt den freien Willen eines Menschen als bloße Illusion erscheinen. Dies ist Herrn Metzinger zu einfach. Kein Hirnforscher könne die Innenperspektive der Fledermaus beschreiben, auch wenn noch so viel Materielles experimentell aufgeklärt sei. Besondere Bedeutung komme der Morallehre zu. Wenn der Mensch durch seine Hirnstrukturen vorprogrammiert sei, verlören moralische Werte ihren absoluten Charakter. Dies hätte weitreichende Folgen für die Gesellschaft. Themen wie Leben, Tod, Schuld, Sühne und Moral wären der Beliebigkeit des physiologischen Hirnzustandes ausgeliefert. In dieser Beziehung sieht er bedenkliche Entwicklungen in unserer Gesellschaft.

Am Samstag wurde die Moderation durch Herrn Heinrich (Solingen) fortgesetzt. Herr Klein (Bochum) berichtete über das "Metabolische Syndrom". Der Begriff "Metabolisches Syndrom" hat sich aus der Diabetologie entwickelt. Er ist gekennzeichnet durch eine Vielzahl von Stoffwechselstörungen auf der Basis einer zum Typ-2Diabetes führenden Insulinresistenz. Weitere Kennzeichen sind die Risikofaktoren Übergewicht, Bluthochdruck und abnorme Blutfettwerte, die meist zu kardiovaskulären Komplikationen führen. Obwohl Insulin in ausreichender Konzentration im Plasma vorhanden ist, nimmt die Zelle keine Glucose auf. Ein erhöhter Blutzukkerspiegel führt zur vermehrten Ausschüttung von Insulin, das nicht mehr angemessen auf die Körperzellen einwirken kann. Genetische Ursachen sind schwer nachweisbar, da genetische Faktoren und Umweltfaktoren zusammenwirken. Bisher glaubte man, dass Typ-2-Diabetes durch einen Defekt an den Insulinrezeptorzellen verursacht wird. Inzwischen ist jedoch das übermäßig vorhandene Fettgewebe in den Blickpunkt des Interesses gerückt. Das Fettgewebe wird gleichsam als "endokrines Organ" bezeichnet. Es schüttet hormonähnliche Substanzen aus wie z.B. Leptin und Adiponectin, die den Energieumsatz steuern. Eine erhöhte Konzentration dieser Substanzen ist mit Bluthochdruck und Insulinresistenz assoziiert. Das metabolische Syndrom ist eine Zivilisationskrankheit. Medikamentös kann man die Progression des Typ-2-Diabetes verhindern (Glitazone). Vorteilhafter ist es jedoch, den Lebensstil und die Essgewohnheiten präventiv zu ändern. Bewegung, angemessene Ernährung und Nikotinverzicht sind die besten Garanten, um das metabolische Syndrom zu vermeiden oder einen Typ-2-Diabetes hinauszuzögern.

Als nächster Referent sprach Herr Hinzmann (Norderstedt) zum Thema "Klinische Bedeutung der Quantifizierung unreifer Thrombozyten". Die Lebensdauer von Thrombozyten beträgt 9-10 Tage. Die vorausgehende Unreife dauert 1-2 Tage. Unreife Thrombozyten enthalten DNA, die mit Thiazolorange angefärbt werden kann. Der Referenzbereich liegt bei 1.1 bis $6.1 \%$, allerdings ist er methodenspezifisch variabel. Differentialdiagnostisch kann mit Kenntnis der unreifen Thrombozyten zwischen verminderter Synthese im Knochenmark und erhöhtem Verbrauch von Thrombozyten in der Peripherie unterschieden werden. Analytisch gibt es zwei Wege: Quantifizierung mittels Durchflusszytometrie mit CD41 und CD61 oder Anfärbung mit einem Farbstoff. Im SysmexSystem werden zwei Farbstoffe auf Polymethin/OxazinBasis verwendet. Studien belegen, dass die unreifen Thrombozyten bei der ITP (idiopathischen Thrombozytopenie) und der TTP (thrombotisch-thrombozytopenischen Purpura) um 100\% erhöht sind. Andere Untersuchungen zielen auf die Möglichkeit ab, Thrombozytenkonzentrate bei der autologen Stammzelltransplantation einzusparen. Weitere Studien sind im Gange.

Nach der Kaffepause berichtete Herr Überla (Bochum) über die HIV-Diagnostik: "HIV - ein Update". Trotz intensiver HIV-Forschung sind die Erfolge bei der Bekämpfung des HIV-Virus begrenzt. Es gibt keine Heilungschancen, es gibt keinen Impfstoff. Fortschritte wurden bei der antiviralen Therapie durch Einsatz von Reverse-Transkriptase-Inhibitoren und Proteasen erzielt. Nach langer Therapie sind allerdings Resistenzentwicklung und Kreuzresistenzen zu beobachten. Die Resistenztestung verschiedener HIV-Populationen kann mittels PCR durchgeführt werden. Durch Vergleich in einer Datenbank kann die Sensitivität bestimmt werden. Unsicherheiten werden jedoch durch Mischpopulationen, unbekannte Mutationen, neue Medikamente und Kreuzresistenzen 
hervorgerufen. Der alternative phänotypische HIV-Resistenztest in der Zellkultur wiederum dauert mit zwei Wochen zu lange. Seine Indikation ist nur bei Therapieversagen gegeben. Interessant, aber nur in Einzelfällen beobachtbar ist das Verschwinden von multiresistenten HIV-Varianten bei Therapiepause. Möglicherweise findet eine Rückmutation statt. Bei der strukturierten Therapiepause spekuliert man auf eine anschließende Boosterung der Immunantwort, nachdem sie durch antivirale Therapie geschwächt worden ist. Bei der Impfstoffentwicklung sind die Anstrengungen sehr groß, allerdings ist ein Durchbruch nicht absehbar. In Bochum wird mit modifizierten HIV-Partikeln experimentiert, die für das Immunsystem besser erkennbar sein sollen. Bei Primaten konnte die Viruslast wirksam gesenkt werden. Bis zum Einsatz am Menschen dürften noch mehrere Jahre vergehen.

Im anschließenden Round-Table-Gespräch beleuchtete Herr Schmitz (Bochum) "Kooperationsformen zwischen Krankenhaus-Laboratorien”. Zunächst zählte er verschiedene Gesellschaftsformen (Personen- oder Kapitalgesellschaften) mit ihren spezifischen Vor- und Nachteilen für ein Kooperationsmodell auf. Die EU bietet eine neue Möglichkeit durch eine Gesellschaftsform nach englischem Recht und Sitzverlegung nach Deutschland. Diese ist zwar kostengünstig, wird aber später durch Bilanzpflichten aufwändiger. Im Krankenhaus wird nicht selten eine $\mathrm{GmbH}$ gebildet. Die Mitarbeiter haben ein Widerspruchsrecht gegen den Übergang ihres Arbeitsverhältnisses in diese Gesellschaftsform, denn ein Jahr danach könnte eine betriebsbedingte Kündigung folgen. Das Ziel im Krankenhauslabor sollte die Erschließung des ambulanten Marktes sein. Eine Möglichkeit dazu bestünde in der Etablierung eines medizinischen Versorgungszentrums, in dem der Laborarzt für die ambulante Versorgung teilzeitbeschäftigt wäre. Dafür sollte es in der Ärzteschaft eine neue Musterberufsordnung geben.

Das Gesundheitssystem steht in zunehmendem Maße im Spannungsfeld zwischen medizinischer Notwendigkeit und wirtschaftlichen Zwängen. Die Vergütung nach Fallpauschalen soll im Rahmen des DRG-Systems (Diagnosis Related Groups-Systems) über Wettbewerb zu stabilen Kosten führen. Die Einführung sollte in einer Konvergenzphase von 2002 bis 2007 erfolgen. Die Gesetzgebung für eine Verlängerung bis 2009 ist fast abgeschlossen. Laborrelevante Befunde sind solche, die zu Nebendiagnosen führen und mit Komplikationen verbunden sind. Solche Befunde können zur Steigerung des Casemix führen, d.h. zu höheren Durchschnittskosten pro Fall für das Krankenhaus. In der Durchführungsphase des DRG-Systems wird es darauf ankommen, die möglichst zum Ziel führenden und nicht nur die billigsten Analysen anzufordern. Eine besondere Bedeutung kommt der Information des Arztes über die Kosten der Analysen zu. Laborintern ist weiter an der Senkung der variablen Kosten und Fixkosten zu arbeiten. Im Krankenhaus müssen die Behandlungsprozesse optimiert werden. Hier sollte das Labor im Sinne eines Kompetenzzentrums Beratung und Qualitätsvorgaben mitliefern.

Anschließend berichtete Herr Kleesiek (Bad Oeynhausen) über "Aktuelles aus dem Fach". Die besonderen Anstrengungen des Vorstandes der DGKL seien darauf ausgerichtet, die Klinische Chemie als selbstständiges Fach in Wissenschaft, Lehre und Krankenversorgung zu erhalten. Hierzu habe der Vorstand der DGKL ein Strategiepapier entwickelt, das bei Herrn Vogt erhältlich sei. Parallel dazu sei ein Rechtsgutachten erstellt worden, das den Anspruch einer wissenschaftlichen Vertretung der Klinischen Chemie an Deutschen Hochschulen begründen soll. Hochschulen ohne wissenschaftlichinstitutionelle Vertretung der Klinischen Chemie verstoßen gegen Grundrechte. Dieses Gutachten soll an Universitäten, Ministerien und Kollegen versandt werden. Strukturveränderungen in der Hochschulmedizin zeichnen sich ab. Stichworte sind: Streichung von Habilitation, befristete Juniorprofessur, Aufhebung des Verbotes der Hausberufung. Bedauerlicherweise würden Stiftungsprofessuren von den Hochschulen abgelehnt. Daneben ändere sich die Laborstruktur im niedergelassenen Bereich und im Krankenhausbereich durch Outsourcing, Insourcing und Kooperationen. Bei all diesen Entwicklungen, nicht zuletzt auch im Bereich der Weiterbildungsordnung, möchte die DGKL gestaltenden Einfluss nehmen, um die Qualität der Leistungserbringung zu erhalten. Die nächste Jahrestagung der DGKL wird vom 6. bis 8. Oktober 2005 in Jena stattfinden.

Das diesjährige Laborleitertreffen war wiederum geprägt von anspruchsvollen Vorträgen, interessanten Diskussionen und der angenehmen Atmosphäre beim persönlichen Gedankenaustausch.

Herr Krieg und Herr Heinrich führten die Moderation in bewährter Weise. Alle Teilnehmer dankten Herrn Krieg, Herrn Heinrich und Herrn Dick für die wiederum gelungene Organisation des Treffens.

Dank und Anerkennung gebührt ebenfalls den Firmen Roche Diagnostics $\mathrm{GmbH}$ und Sysmex Deutschland $\mathrm{GmbH}$, die das Laborleitertreffen großzügig unterstützt haben. 